\title{
Mobility Method Applied to Calculate the Lubrication Properties of Bearing under Dynamic Loads
}

\author{
Tao He, Xiqun Lu, and Jingzhi Zhu \\ College of Power and Energy Engineering, Harbin Engineering University, Harbin, \\ Heilongjiang 150001, China
}

Correspondence should be addressed to Xiqun Lu; luxiqun@gmail.com

Received 29 November 2012; Accepted 24 December 2012

Academic Editors: B. Chan and B. Yu

Copyright (C) 2013 Tao He et al. This is an open access article distributed under the Creative Commons Attribution License, which permits unrestricted use, distribution, and reproduction in any medium, provided the original work is properly cited.

The analytical mobility method for dynamically loaded journal bearings was presented, with the intent to include it in a general computational program, such as the dynamic analysis program, that has been developed for the dynamic analysis of general mechanical systems. An illustrative example and numerical results were presented, with the efficiency of the method being discussed in the process of their presentation.

\section{Introduction}

The mobility method is widely used for analyzing rigid journal bearings due to its computational efficiency when compared to other approaches such as the multigrid techniques based on the Elrod algorithm and the finite element methods of analysis [1-8]. It is still a mainstay of engine bearing performance calculation because of its rapid prediction ability even though many other comprehensive methods have been developed for complicated bearing analysis. In fact, the mobility method is sufficiently accurate and easy to understand and apply by designers and engineers that do not need to be expert in the field of tribology.

\section{Model}

The mobility method introduced by Booker [1] is a technique for direct evaluation of the instantaneous velocity of the journal center in terms of the applied load and the bearing geometry. By determining the velocity of the bearing center, the orbits of the journal center can be obtained.
From [2], the Reynolds equation for a dynamically loaded journal bearing with an isothermal lubrication in the polar coordinate system can be written as

$$
\begin{aligned}
& \frac{\partial}{\partial \theta}\left[h^{3} \frac{\partial p}{\partial \theta}\right]+R^{2} \frac{\partial}{\partial z}\left[h^{3} \frac{\partial p}{\partial z}\right] \\
& =12 \mu R^{2} c[\dot{\varepsilon} \cos \theta+\varepsilon(\dot{\varphi}-\bar{\omega}) \sin \theta],
\end{aligned}
$$

where $\theta$ and $z$ are the bearing coordinates, $\mu$ is the dynamic oil viscosity, $h$ is the oil film thickness at time $t$, and $c$ is the radial clearance; $\bar{\omega}=\left(\omega_{j}+\omega_{b}\right) / 2-\dot{\psi}$ is the average angular velocity of journal and bearing relative to the external load vector $F(t), \omega_{j}$ is the angular velocity of the journal, $\omega_{b}$ is the angular velocity of the bearing, and $\dot{\psi}$ is the angular velocity of the load; $\dot{\varphi}=d \varphi / d t$ is the angular velocity of the attitude angle, and $\varphi$ is the attitude angle, that is, an angle between the applied load direction and the center line of the journal and the bearing; $\dot{\varepsilon}$ is the radial velocity of the journal center, and $\varepsilon \dot{\varphi}$ is the tangential velocity of the journal center.

The geometry of a dynamically loaded journal bearing and its notations are shown in Figure 1, for the purpose of defining the mobility. 


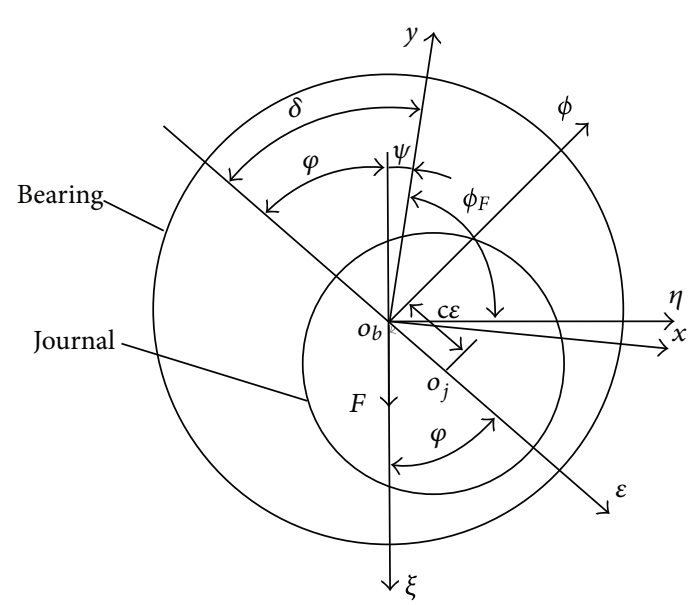

FIGURE 1: Journal bearing geometry for dynamic loading.

The right hand side of (1) includes unknowns $\dot{\varepsilon}$ and $\dot{\varphi}$. These two unknowns can be determined by integrating the trajectory of the journal center. If the pressure distribution is known, the load carrying capacity components can be obtained to balance the applied load, and the balance can be written as

$$
\begin{aligned}
& F^{\varepsilon}=F \cos \varphi=-\int_{0}^{L} \int_{\theta_{1}}^{\theta_{2}} p \cos \theta d \theta d z, \\
& F^{\varphi}=-F \sin \varphi=\int_{0}^{L} \int_{\theta_{1}}^{\theta_{2}} p \sin \theta d \theta d z .
\end{aligned}
$$

The positive portion of the hydrodynamic pressure distribution lies between $\theta_{1}$ and $\theta_{2}$, given by the following.

For short bearing,

$$
\begin{gathered}
\theta_{1}=\tan ^{-1} \frac{\dot{\varepsilon}}{\varepsilon(\bar{\omega}-\dot{\varphi})}, \\
\theta_{2}= \begin{cases}\theta_{1}+\pi, & \text { when } \varepsilon(\bar{\omega}-\dot{\varphi})>0, \\
\theta_{1}-\pi, & \text { when } \varepsilon(\bar{\omega}-\dot{\varphi})>0 .\end{cases}
\end{gathered}
$$

For infinitely long bearing,

$$
\begin{gathered}
\theta_{1}=\tan ^{-1} \frac{\left(2+\varepsilon^{2}\right) \dot{\varepsilon}}{2 \varepsilon(\bar{\omega}-\dot{\varphi})}, \\
\theta_{2}= \begin{cases}\theta_{1}+\pi, & \text { when } \varepsilon(\bar{\omega}-\dot{\varphi})>0, \\
\theta_{1}-\pi, & \text { when } \varepsilon(\bar{\omega}-\dot{\varphi})>0 .\end{cases}
\end{gathered}
$$

It is important to note that angles $\theta_{1}$ and $\theta_{2}$ change with the work conditions of journal bearing. Thus, for each instant of simulation, the pressure is function of the journal bearing geometric properties, its kinematic characteristics, and varies in both circumferential and axial directions.

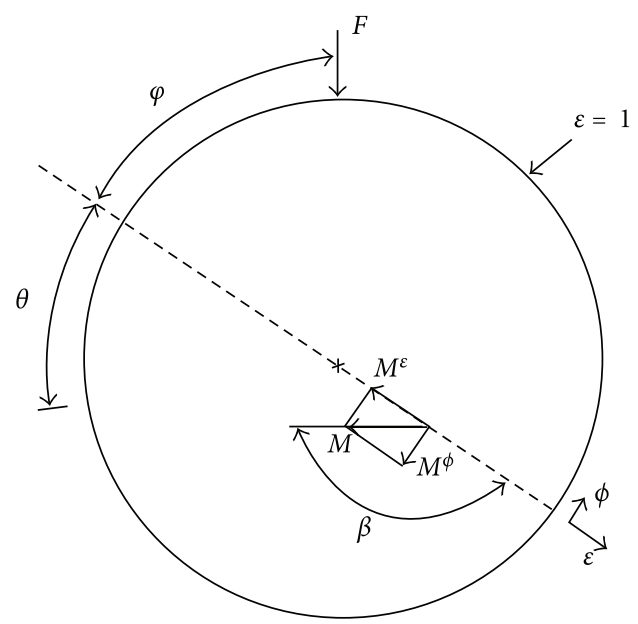

FIGURE 2: Representation of mobility vector and its components.

In [3], in order to simplify the solution of Reynolds' equation, Booker proposed to express the $\dot{\varepsilon}$ and $\dot{\varphi}$ as function of mobility components as

$$
\begin{gathered}
\dot{\varepsilon}=\frac{F(c / R)^{2}}{\mu L D} M^{\varepsilon}, \\
\dot{\varphi}=\frac{F(c / R)^{2}}{\mu L D \varepsilon} M^{\phi}+\bar{\omega} .
\end{gathered}
$$

In [4], these two functions, $M^{\varepsilon}$ and $M^{\phi}$, can be considered as the components of a mobility vector $\vec{M}$, which is defined as a ratio of velocity to force. If $\beta$ is mobility direction angle, as it is illustrated in Figure 2, we may write

$$
M^{\varepsilon}=-\vec{M} \cos \beta, \quad M^{\varphi}=-\vec{M} \sin \beta .
$$

$\vec{M}$ is a function only of the position vector $\vec{\varepsilon}$ and the ratio of $L / D$. The components of the mobility vector, $\vec{M}(\varepsilon, L / D)$, that is, $M^{\varepsilon}$ and $M^{\phi}$ can be computed numerically. If mobility data for a particular bearing is available, it is relatively simple to solve (1). Substituting (5) into (1), we can obtain

$$
\begin{aligned}
& \frac{\partial}{\partial \theta}\left[h^{3} \frac{\partial p}{\partial \theta}\right]+R^{2} \frac{\partial}{\partial z}\left[h^{3} \frac{\partial p}{\partial z}\right] \\
& \quad=12 \mu R^{2} c\left[\frac{F(c / R)^{2}}{\mu L D} M^{\varepsilon} \cos \theta+\varepsilon\left(\frac{F(c / R)^{2}}{\mu L D \varepsilon} M^{\phi}\right) \sin \theta\right] .
\end{aligned}
$$

Equation (7) can be solved numerically and new pressure distribution is thus obtained. Bearing load capacity can be calculated to balance the applied load.

The above discussion can lead to a procedure for the use of the mobility method to analyze dynamically loaded journal bearings. When the ratio of bearing length to bearing diameter $(L / D)$ is less than or equal to $0.75[5]$, a bearing can be assumed to act as a short bearing. The short bearing assumption is used to eliminate the pressure term in the circumferential direction from Reynolds equation, that is, 
the first term on the left hand side of (7). Because of this assumption, $(\partial / \partial \theta)\left[h^{3}(\partial p / \partial \theta)\right] \ll R^{2}(\partial / \partial z)\left[h^{3}(\partial p / \partial z)\right]$. Hence, Reynolds equation can be reduced to:

$$
\begin{aligned}
& R^{2} \frac{\partial}{\partial z}\left[h^{3} \frac{\partial p}{\partial z}\right] \\
& \quad=12 \mu R^{2} c\left[\frac{F(c / R)^{2}}{\mu L D} M^{\varepsilon} \cos \theta+\varepsilon\left(\frac{F(c / R)^{2}}{\mu L D \varepsilon} M^{\phi}\right) \sin \theta\right] .
\end{aligned}
$$

By integrating (8) twice, the hydrodynamic pressure along the $z$ axis is obtained as

$$
\begin{aligned}
p= & \frac{6 \mu c}{h^{3}}\left[\frac{F(c / R)^{2}}{\mu L D} M^{\varepsilon} \cos \theta+\varepsilon\left(\frac{F(c / R)^{2}}{\mu L D \varepsilon} M^{\phi}\right) \sin \theta\right] \\
& \times\left[z^{2}+2 c_{1} z+c_{2}\right] .
\end{aligned}
$$

Using the boundary condition that $p(\theta, \pm L / 2)=0$, we can evaluate the constants in (9): $c_{1}=0, c_{2}=-L^{2} / 4$. Therefore, the hydrodynamic pressure is

$$
\begin{aligned}
p= & \frac{6 \mu c}{h^{3}}\left[\frac{F(c / R)^{2}}{\mu L D} M^{\varepsilon} \cos \theta+\varepsilon\left(\frac{F(c / R)^{2}}{\mu L D \varepsilon} M^{\phi}\right) \sin \theta\right] \\
& \times\left[z^{2}-\frac{L^{2}}{4}\right] .
\end{aligned}
$$

Substituting (10) into (2) leads to

$$
\begin{aligned}
F^{\varepsilon}= & -\int_{0}^{L} \int_{\theta_{1}}^{\theta_{2}} p(\theta, z) \cos \theta d \theta d z \\
= & \int_{\theta_{1}}^{\theta_{2}} \frac{\mu L^{3} R}{c^{2}}\left[\frac{F(c / R)^{2}}{\mu L D} M^{\varepsilon} \cos \theta+\varepsilon\left(\frac{F(c / R)^{2}}{\mu L D \varepsilon} M^{\phi}\right) \sin \theta\right] \\
& \times \frac{\cos \theta}{(1+\varepsilon \cos \theta)^{3}} d \theta, \\
F^{\varphi}= & \int_{0}^{L} \int_{\theta_{1}}^{\theta_{2}} p(\theta, z) \sin \theta d \theta d z \\
= & \int_{\theta_{1}}^{\theta_{2}} \frac{\mu L^{3} R}{c^{2}}\left[\frac{F(c / R)^{2}}{\mu L D} M^{\varepsilon} \cos \theta+\varepsilon\left(\frac{F(c / R)^{2}}{\mu L D \varepsilon} M^{\phi}\right) \sin \theta\right] \\
& \times \frac{\sin \theta}{(1+\varepsilon \cos \theta)^{3}} d \theta .
\end{aligned}
$$

Solving (2) and (11) simultaneously produces

$$
\begin{aligned}
M^{\varepsilon} & =\frac{\left(I_{3}^{20} \cos \varphi+I_{3}^{11} \sin \varphi\right)}{2(L / D)^{2}\left(I_{3}^{20} I_{3}^{02}-I_{3}^{11} I_{3}^{11}\right)}, \\
M^{\phi} & =\frac{\left(I_{3}^{02} \cos \varphi+I_{3}^{11} \sin \varphi\right)}{2(L / D)^{2}\left(I_{3}^{20} I_{3}^{02}-I_{3}^{11} I_{3}^{11}\right)},
\end{aligned}
$$

where $I_{n}^{l m}=\int_{\theta}\left(\sin ^{l} \theta \cos ^{m} \theta /(1+\varepsilon \cos \theta)^{n}\right) d \theta$.
The solution of the above equations is complex and involves a good deal of mathematical manipulation. However, Booker [1] gave the numerical solution to (12), for positive force, $F>0$,

$$
\begin{gathered}
M^{\varsigma}=\frac{(1-\varsigma)^{5 / 2}}{\pi(L / D)^{2}}, \\
M^{\kappa}=-\frac{4 \kappa(1-\varsigma)^{3 / 2}}{\pi^{2}(L / D)^{2}}
\end{gathered}
$$

and, for negative force, $F<0$,

$$
\begin{gathered}
M^{\varsigma}=\frac{(1+\varsigma)^{5 / 2}}{\pi(L / D)^{2}}, \\
M^{\kappa}=\frac{4 \kappa(1+\varsigma)^{3 / 2}}{\pi^{2}(L / D)^{2}}
\end{gathered}
$$

with

$$
\begin{gathered}
M^{\varepsilon}=M^{\varsigma} \cos \varphi+M^{\kappa} \sin \varphi, \\
M^{\varphi}=-M^{\varsigma} \sin \varphi+M^{\kappa} \cos \varphi, \\
\varsigma=\varepsilon \cos \varphi, \\
\kappa=\varepsilon \sin \varphi .
\end{gathered}
$$

For a specified external load $F(t),(13)-(15)$ are solved for $\dot{\varepsilon}$ and $\dot{\varphi}$ and then integrated with respect to time, in order to obtain the journal center trajectory, that is, $\varepsilon(t), \varphi(t)$.

Similarly, the above scheme can be applied to infinitely long bearings. But for a bearing of finite length, both hydrodynamic pressure terms in the Reynolds equation are significant and cannot be neglected in the calculation of the pressure distribution. However, no closed form analytical exists for (7). Therefore, numerical methods are extensively used to solve (7). From a vast number of solutions obtained bearing hydrodynamic lubrication problems, the mobility components can be expressed in a closed formation using a curve fit approach, see, for example, Goenka [6]. When the ratio of bearing length to bearing diameter ranges between 0.75 and 4 , a bearing is assumed to be a finite length bearing.

\section{Numerical Procedure}

With a knowledge of the hydrodynamic pressure distribution, the load carrying capacity can be determined and $(\dot{\varepsilon}, \dot{\varphi})$ can be modified to ensure the applied load is balanced. When the balance is achieved, with the knowledge of the journal center velocity, journal movement over a time step can be determined. A new film profile may thus be obtained. A new pressure distribution for the new instantaneous operating conditions is then required, and the process is repeated until convergence is obtained. A computational scheme for using the mobility method in the analysis of a bearing is as follows.

(1) Make an initial guess for $\varepsilon, \varphi, \dot{\varepsilon}, \dot{\varphi}$.

(2) Determine $\theta_{1}$ and $\theta_{2}$, then obtain the pressure distribution. 
TABLE 1: Bearing characteristics.

\begin{tabular}{lc}
\hline Journal bearing length & $0.11 \mathrm{~m}$ \\
Journal bearing diameter & $0.23 \mathrm{~m}$ \\
Fluid viscosity $(313 \mathrm{~K})$ & $135 \sim 165 \mathrm{~mm}^{2} / \mathrm{s}$ \\
Radial clearance & $4 e-5 \mathrm{~m}$ \\
Speed & $1050 \mathrm{rpm}$ \\
Initial eccentricity, $\varepsilon^{0}$ & 0.1 \\
Initial attitude angle, $\varphi^{0}$ & $1.5 \mathrm{rad}$ \\
Time step & $0.1 \mathrm{deg}$ \\
\hline
\end{tabular}

(3) Calculate $\dot{\varepsilon}$ and $\dot{\varphi}$ using mobility data.

(4) Evaluate $\varepsilon, \varphi$ at next time step by using $\varepsilon^{t+1}=\varepsilon^{t}+\dot{\varepsilon} \Delta t$, $\varphi^{t+1}=\varphi^{t}+\dot{\varphi} \Delta t$.

(5) Repeat steps (2)-(4) until the last time step in one engine cycle.

(6) Check for the convergence of $\varepsilon$ and $\varphi$ between the values of the initial guess and the last time step.

(7) Set the values of the last time step as new guess of $\varepsilon, \varphi, \dot{\varepsilon}, \dot{\varphi}$, and repeat steps (2) to (6) if the results have not converged.

\section{Results and Discussion}

A rigid bearing is analyzed using the scheme developed in above. The related bearing parameters are listed in Table 1, and Figure 3 shows the diagram of the dynamic load applied to the bearing during one engine cycle. For a four-stroke engine, there are 720 degree crank angle in one engine cycle. The time step corresponding to the analysis is one degree crank angle; thus, there are 720 time-steps in one engine cycle. When the engine speed is $1050 \mathrm{rpm}$, the total time corresponding to one engine cycle is $720^{\circ} \times 60 / 1050 \times 360 \approx$ $0.1143 \mathrm{~s}$. The time corresponding to one degree crank angle is $1.59 \times 10^{-4} \mathrm{~s}$. The bearing is divided into a grid $\left(N_{x} \times N_{z}\right)$ with a grid size $\Delta x \times \Delta z$. A grid size with $37 \times 21$ grid points is used in the program code.

It should be noted that, with this approach of mobility method, the choice of the initial conditions does not affect the relative journal bearing motion and only influences the first instants of simulation. Thus, the cyclic behavior of the journal, independent of the assumed initial values, is reached very quickly. In Figure 4 the journal eccentricity in $x$ and $y$ direction is shown as function of the crank angle. In dynamically loaded journal bearings both the eccentricity and attitude angle vary with the cycle of applied load, and the correct design should ensure that the combination of load and speed rotation does not lead a dangerous small minimum film thickness.

The minimum film thickness is related to the eccentricity ratio and radial clearance as follows:

$$
h_{\min }=c(1-\varepsilon) .
$$

The variation of the minimum film thickness during the dynamic simulation of journal bearing gives the value of

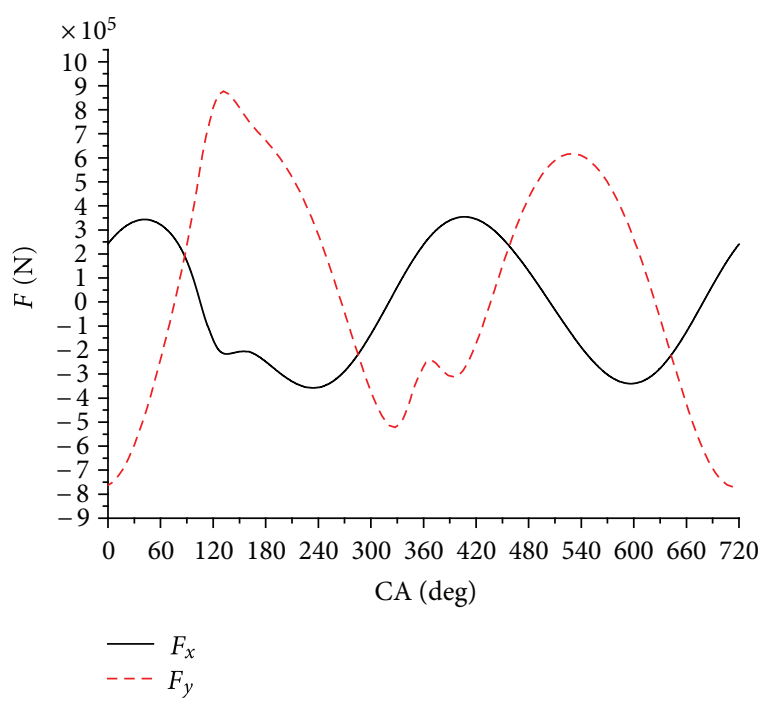

Figure 3: The bearing load.

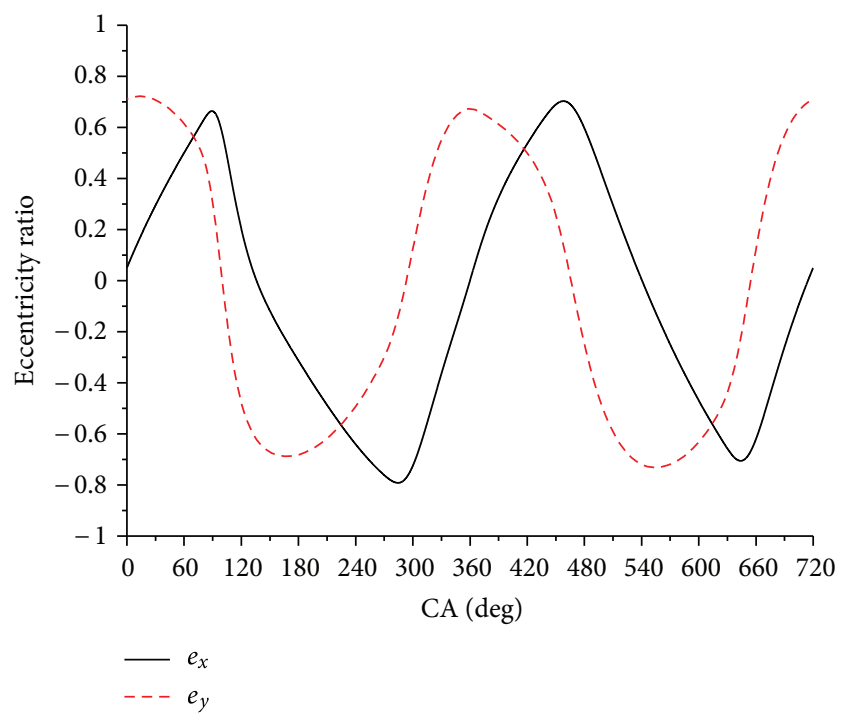

FIGURE 4: Journal center eccentricity.

the absolute minimum film thickness. This value should be greater than the average asperity height of the journal bearing surfaces in order to prevent the metal-to-metal contact and, consequently, to avoid the friction and wear, besides keeping the hydrodynamic approach valid. From the journal bearing design view point, the safe value for film thickness is $2.5 \mu \mathrm{m}$, and, in practical, the minimum film thickness should be at least $(1.0-1.5) \times 2.5 \mu \mathrm{m}$ [7]. Figure 5 shows the variation of the minimum film thickness with the angle. The dashed horizontal line in the same figure represents that safe film thickness. By observing Figure 5, it is clear that the effective hydrodynamic lubrication is performed on the journal bearing, meaning that the journal and bearing surfaces are completely separated. 


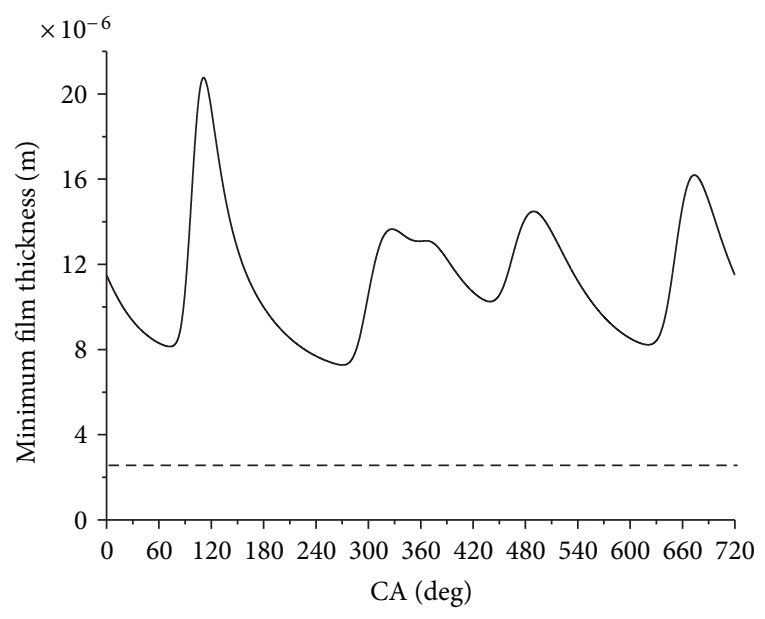

FIGURE 5: The minimum film thickness.

\section{Acknowledgments}

The present research has been funded by National Natural Science Foundation of China (Grant no. 50979016), Program for New Century Excellent Talents in University of Ministry of Education of China, and Special Fund for Basic Research Program of Higher Education of China (Code: HEUCF100308). The authors would like to sincerely express their appreciation.

\section{References}

[1] J. F. Booker, "Dynamically loaded journal bearings: numerical application of mobility method," Journal of Lubrication Technology, vol. 1, pp. 168-176, 1971.

[2] P. Flires, J. C. P. Claro, and J. Ambrosio, "Journal bearings subjected to dynamic loads: the analytical mobility method," Mecanica Experimental, vol. 13, pp. 115-127, 2006.

[3] W. E. Ten Napel, H. Moes, and R. Bosma, "Dynamically loaded pivoted pad journal bearings: mobility method of solution," Journal of Lubrication Technology, vol. 98, no. 2, pp. 196-205, 1976.

[4] J. Frene, D. Nicolas, B. Degeurce, D. Berthe, and M. Godet, Hydrodynamic Lubrication: Bearings and Thrust Bearings, Elsevier, Amsterdam, The Netherlands, 1997.

[5] G. B. Dubois and F. W. Ocvirk, "Analytical derivation and experimental evaluation of short-bearing approximation for full journal bearings," NACA Technical Report 1157, 1953.

[6] P. K. Goenka, "Analytical curve fits for solution parameters of dynamically loaded journal bearings," Journal of Tribology, Transactions of the ASME, vol. 106, no. 4, pp. 421-428, 1984.

[7] B. J. Hamrock, Fundamentals of Fluid Film Lubrication, McGraw-Hill, New York, NY, USA, 1994.

[8] B. Vincent, P. Maspeyrot, and J. Frene, "Cavitation in dynamically loaded journal bearings using mobility method," Wear, vol. 193, no. 2, pp. 155-162, 1996. 

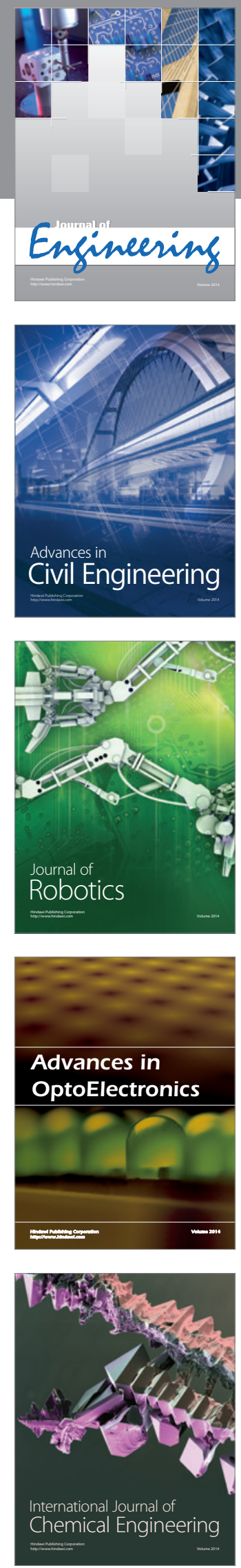

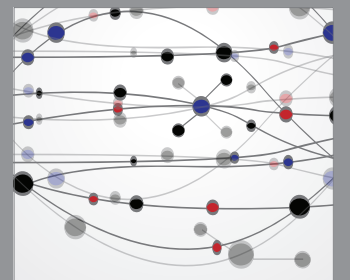

The Scientific World Journal
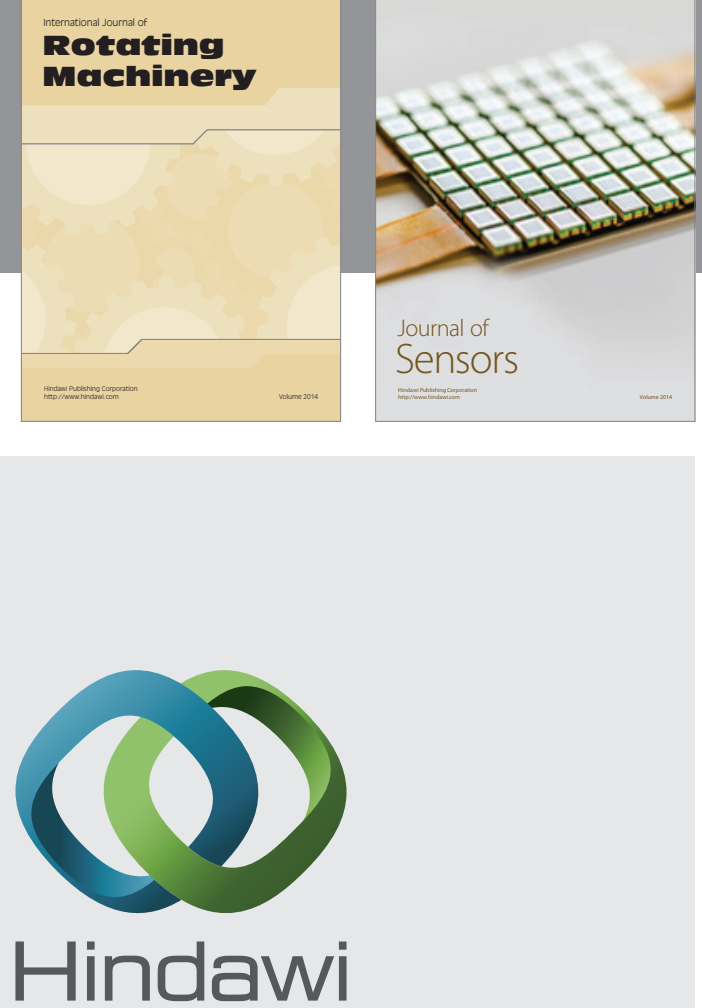

Submit your manuscripts at http://www.hindawi.com
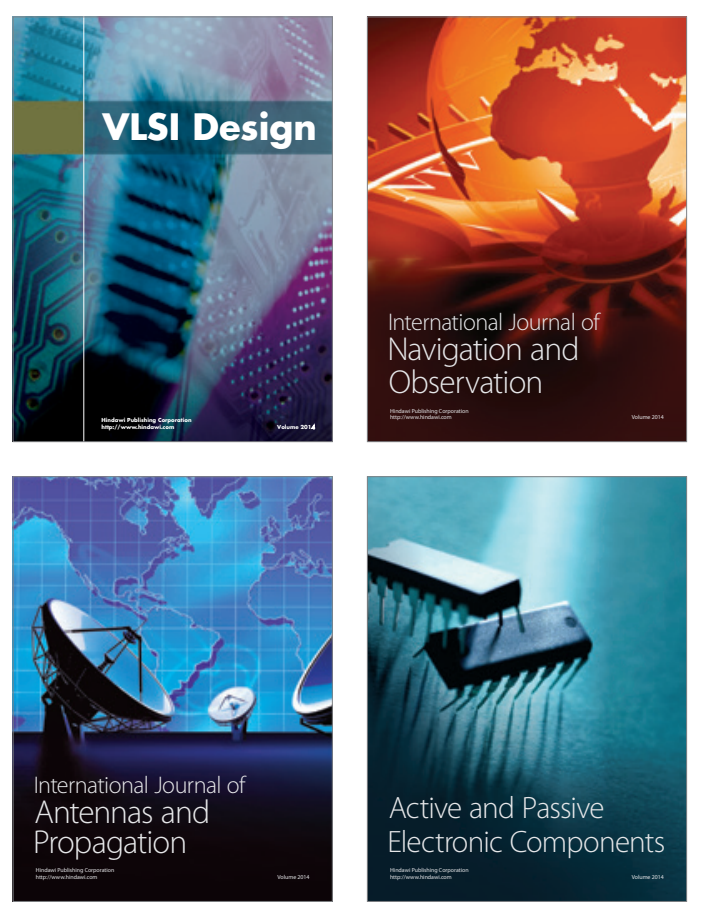
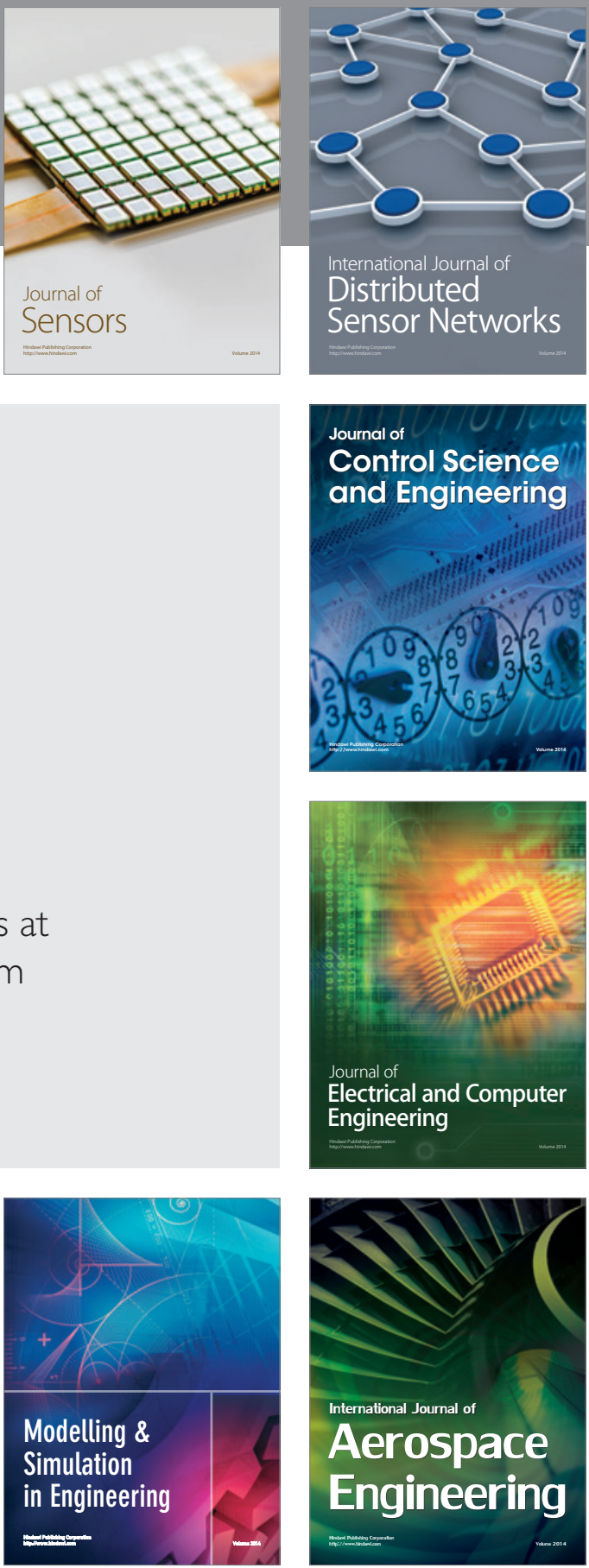

Journal of

Control Science

and Engineering
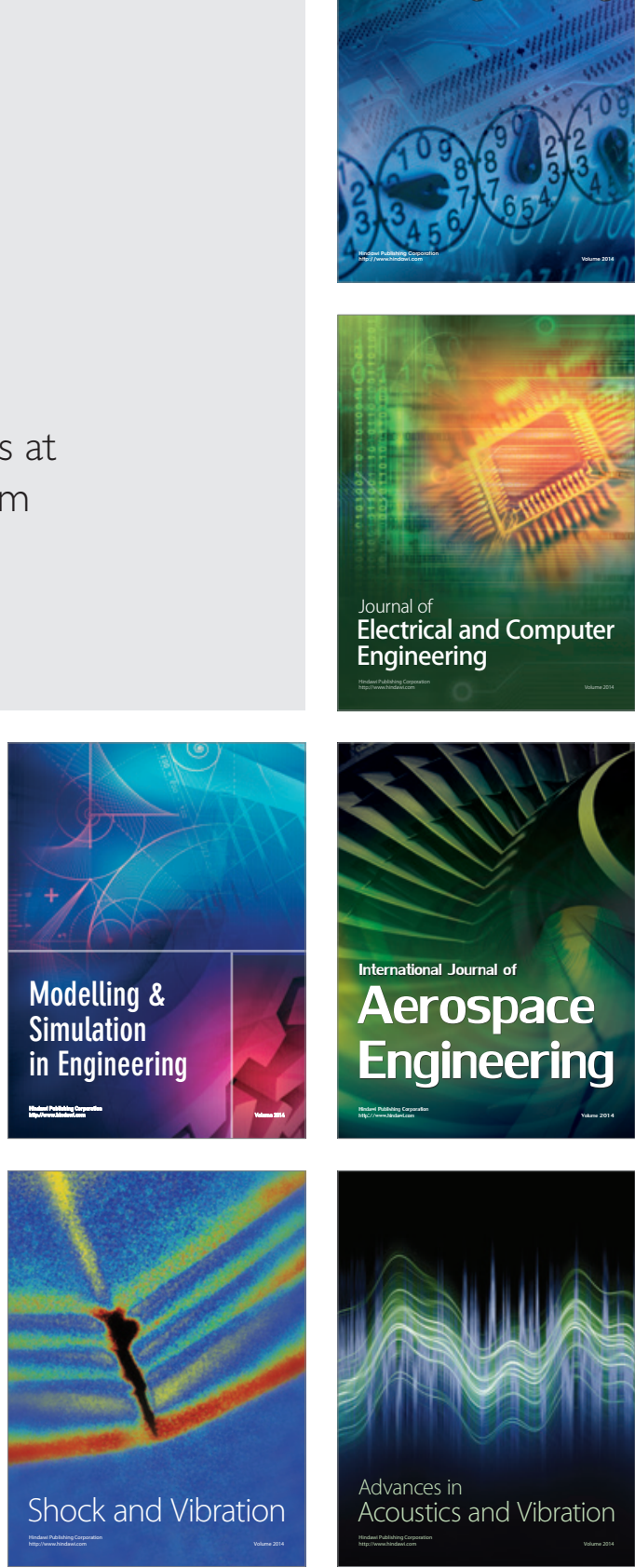